\title{
Association of COMT and COMT-DRD2 interaction with creative potential
}

\section{Shun Zhang, Muzi Zhang and Jinghuan Zhang*}

Department of Psychology, Shandong Normal University, Jinan, China

\section{Edited by:}

Zbigniew R. Struzik, The University of Tokyo, Japan

Reviewed by:

Bernhard Hommel, Leiden

University, Netherlands

Adam E. Green, Georgetown

University, USA

*Correspondence:

Jinghuan Zhang, Department of

Psychology, Shandong Normal

University, No. 88 East Wenhua

Road, Jinan, Shandong,

250014, China

e-mail: zhangjinghuan@sdnu.edu.cn
Several lines of evidence suggest that genes involved in dopamine (DA) transmission may contribute to creativity. Among these genes, the catechol-O-methyltransferase gene (COMT) and the dopamine D2 receptor gene (DRD2) are the most promising candidates. Our previous study has revealed evidence for the involvement of DRD2 in creative potential. The present study extended our previous study by systematically exploring the association of COMT with creative potential as well as the interaction between COMT and DRD2. Twelve single nucleotide polymorphisms (SNPs) covering COMT were genotyped in 543 healthy Chinese college students whose creative potentials were assessed by divergent thinking tests. Single SNP analysis showed that rs 174697 was nominally associated with verbal originality, two SNPs (rs737865 and rs5993883) were nominally associated with figural fluency, and two SNPs (rs737865 and rs4680) were nominally associated with figural originality. Haplotype analysis showed that, the TCT and CCT haplotype (rs737865-rs174675-rs5993882) were nominally associated with figural originality, and the TATGCAG and CGCGGGA haplotype (rs4646312-rs6269-rs4633-rs6267-rs4818-rs4680-rs769224) were nominally associated with figural originality and verbal flexibility, respectively. However, none of these nominal findings survived correction for multiple testing. Gene-gene interaction analysis identified one significant four-way interaction of rs174675 (COMT), rs174697 (COMT), rs1076560 (DRD2), and rs4436578 (DRD2) on verbal fluency, one significant four-way interaction of rs174675 (COMT), rs4818 (COMT), rs1076560 (DRD2), and rs4648317 (DRD2) on verbal flexibility, and one significant three-way interaction of rs5993883 (COMT), rs4648319 (DRD2), and rs4648317 (DRD2) on figural flexibility. In conclusion, the present study provides nominal evidence for the involvement of COMT in creative potential and suggests that DA related genes may act in coordination to contribute to creativity.

Keywords: creativity, creative potential, divergent thinking, dopamine, COMT, DRD2, gene-gene interaction

\section{INTRODUCTION}

Creativity refers to the ability to produce something that is both novel and useful (Sternberg and Lubart, 1999). It is closely related to human development and achievement at both the individual and societal level. Despite its importance, the underlying mechanism of how creativity works is not completely understood. The need for a deeper understanding of the biological correlates of creative cognition has inspired a great number of neuroscience and cognitive studies. Findings from these studies generally support a critical involvement of dopamine (DA) transmission in the cognitive process of creativity (Flaherty, 2005; Chermahini and Hommel, 2010; Takeuchi et al., 2010). Hence, genes involved in DA transmission have been of particular interest to explain individual differences in creativity. Amongst these genes, the catechol-O-methyltransferase gene (COMT) and the dopamine D2 receptor gene (DRD2) have been studied most extensively.

The COMT gene is located on chromosome 22q11. The enzyme encoded by this gene is involved in the inactivation of the catecholamine neurotransmitters (DA, adrenalin, and noradrenalin) (Axelrod, 1957) and is the main factor controlling DA levels in the prefrontal cortex (PFC). The DRD2 gene, located on chromosome 11q22-23, encodes one of five DA receptors and plays an important role in mediating synaptic DA signaling. Variants of these two genes have been repeatedly implicated in creativity related cognitive functions, such as working memory and cognitive control (Egan et al., 2001; Bruder et al., 2005; Zhang et al., 2007; Diaz-Asper et al., 2008; Bertolino et al., 2010; Colzato et al., 2010, 2013).

By employing divergent thinking (DT) tests as a measure of creative potential, several attempts have been made to identify COMT and DRD2 related genetic variants associated with creativity. Reuter et al. (2006) investigated the influence of COMT VAL158MET polymorphism (rs4680) and DRD2/ANKK1 Taq IA polymorphism (rs1800497) on creative potential, and demonstrated that DRD2/ANKK1 rs1800497 was associated with total creativity score. Runco et al. (2011) further extended Reuter et al.'s work by investigating the effects of COMT rs4680 and DRD2/ANKK1 rs1800497 on the three common indexes (fluency, originality, and flexibility) of both verbal and figural DT tests. However, the result indicated that only COMT rs4680 was associated with fluency, and neither of these two genetic variants was related to originality or flexibility when controlling for 
the significant effect of fluency. Although these studies provide important insight into the underlying genetic basis of creativity, it is important to note that, for both COMT and DRD2, only one genetic variant from each gene was investigated. Thus, it is not clear whether there are other genetic variants of these two genes associated with creative potential.

To fill this gap in the literature, our recent study comprehensively explored the associations of DRD2 related genetic polymorphisms with creative potential in the Han Chinese population and found several previously unrevealed DRD2 SNPs and haplotypes associated with DT fluency, originality and flexibility (Zhang et al., 2014). This suggests that a more detailed examination of the genetic variants covering these genes will provide additional valuable information about the effects of these genes on creative potential. Therefore, by using the same approach in the same sample, the present study aimed to systematically investigate the associations of COMT related genetic polymorphisms with creative potential.

Furthermore, there is evidence suggesting a nonlinear relationship between DA and creative potential (Chermahini and Hommel, 2010, 2012). This indicates that interactions among DA related genes may contribute to creativity potential. By reanalyzing Runco et al.'s data, Murphy et al. (2013) recently investigated the interaction between COMT and DRD2. However, because no genetic variant was further genotyped, Murphy et al.'s analysis was confined to the interaction between COMT rs4680 and DRD2/ANKK1 rs1800497, the interaction between COMT and $D R D 2$ on creative potential remains largely unknown and needs to be further assessed. Thus, the present study further extended the literature as well as our previous study by systematically exploring the interaction between COMT and DRD2.

\section{METHODS}

\section{PARTICIPANTS AND PROCEDURE}

The main characteristics of participants and study procedure have been described previously (Zhang et al., 2014). In summary, the sample consisted of 543 unrelated healthy Chinese college students (185 males and 358 females, with a mean age of 18.94 years, $S D=0.84$ ) from Shandong Normal University. All participants were of Han Chinese descendants and with no self-reported history of neurological and psychiatric disorder. This study was approved by the Institutional Review Board of Shandong Normal University, and all study participants gave written informed consent. Participants first completed the psychometric tests, and then peripheral venous blood samples were collected for genotyping.

\section{SNP SELECTION}

In order to ensure a full genetic coverage of COMT, nine tag SNPs (rs737865, rs174675, rs5993882, rs5993883, rs4646312, rs6267, rs4680, rs769224, and rs174697) were selected from HapMap (http://hapmap.ncbi.nlm.nih.gov) genotype data for the $\mathrm{CHB}$ population (Data Rel 27 Phase II + III, Feb09, on NCBI B36 assembly, dbSNP b126) by applying the Tagger program implemented in Haploview v4.2 software (Barrett et al., 2005) with parameters of minor allele frequency (MAF) $>5 \%$ and pairwise $r^{2}>0.8$. The nine tag SNPs captured $80 \%$ of common alleles (MAF $>5 \%$ ) within the genomic region of COMT (chr22:18309309..18336528, based on NCBI Genome Build 36.3) with a mean maximal $r^{2}$ of 0.966 . In addition to the nine tag SNPs, three putative functional SNPs (rs6269, rs4633, and rs4818), all of which were at coding regions, were also genotyped. Table 1 summarizes the final set of genotyped COMT SNPs. The selection of DRD2 SNPs has been described in detail previously (Zhang et al., 2014).

\section{GENOTYPING}

Methods for DNA extraction and genotyping have been described previously (Zhang et al., 2014). Briefly, genomic DNA was extracted from peripheral venous blood sample using the QIAamp DNA Mini Kit (Qiagen, Valencia, CA, USA). Genotypings for all SNPs were performed at Beijing Genomics Institute-Shenzhen (BGI-Shenzhen, CityShenzhen, China) by using the Sequenom ${ }^{\circledR}$ MassARRAY ${ }^{\circledR}$ iPLEX system (Sequenom, San Diego, StateCA, USA). For quality control, 5\% random DNA samples were genotyped twice for each SNP to calculate genotyping error. The genotyping accuracy was 100\%.

\section{CREATIVE POTENTIAL MEASURES—DT TESTS}

As previously described (Zhang et al., 2014), verbal and figural DT tests (each containing three tasks) selected from Runco Creativity Assessment Battery (rCAB; Creativity Testing Service, Bishop, GA, USA) were used to assess creative potential. The $\mathrm{rCAB}$ is comparable to other assessments of fluency, originality, and flexibility (e.g., Wallach and Kogan, 1965). In verbal DT test, participants were asked to list as many different uses as they could for three common subjects (toothbrush, tire, and spoon). In figural DT test, three line drawings were represented and participants were asked to list as many things as each line drawing could be. Four minutes was allowed for each task. All DT tasks were scored for fluency, flexibility, and originality according to the guideline of Creativity Testing Service. Briefly, fluency score was obtained by counting the number of unduplicated responses given by each participant. Originality score was calculated by counting the number of unusual responses (responses given by less than $5 \%$ of the sample). In order to score flexibility, a category list was first generated for each task, and the flexibility score was the number of different categories used in one participant's responses. For each task, two trained raters (both were psychology graduate students from Shandong Normal University) were engaged to score all the responses. The inter-rater reliabilities for all the six DT scores were higher than 0.95 .

\section{STATISTICAL ANALYSIS}

To adjust for confounding factors and the effect of fluency (Hocevar, 1980; Runco and Albert, 1985), covariate-adjusted standardized residuals of DT scores were first calculated using multivariate linear regression. Specifically, for verbal and figural fluency, the covariate adjusted was gender. For verbal originality and flexibility, the covariates included gender and verbal fluency score. For figural originality and flexibility, the covariates included gender and figural fluency score. These residuals were used in association analysis described below.

Hardy-Weinberg equilibrium was tested by Fisher's exact test using Plink v1.07 software (Purcell et al., 2007). Single SNP 
Table 1 | Descriptive statistics and inter-correlations ${ }^{\mathrm{a}}$.

\begin{tabular}{|c|c|c|c|c|c|c|c|c|c|c|}
\hline \multirow[t]{2}{*}{ Measure } & \multirow[t]{2}{*}{ Cronbach's $\alpha$} & \multicolumn{3}{|c|}{$\mathrm{M}(S D)$} & \multirow[t]{2}{*}{1} & \multirow[t]{2}{*}{2} & \multirow[t]{2}{*}{3} & \multirow[t]{2}{*}{4} & \multirow[t]{2}{*}{5} & \multirow[t]{2}{*}{6} \\
\hline & & Total & Male & Female & & & & & & \\
\hline 1. Verbal fluency & 0.85 & $8.25(2.86)$ & $7.43(2.80)$ & $8.67(2.80)^{* *}$ & & $0.84 * *$ & $0.78 * *$ & $0.73^{* *}$ & $0.69 * *$ & $0.60 * *$ \\
\hline 2. Verbal originality & 0.75 & $2.48(1.68)$ & $2.27(1.71)$ & $2.59(1.65)^{*}$ & & & $0.65 * *$ & $0.62 * *$ & $0.63^{* *}$ & $0.51 * *$ \\
\hline 3. Verbal flexibility & 0.70 & $4.40(1.08)$ & $4.25(1.21)$ & $4.48(1.00)^{*}$ & & & & $0.59 * *$ & $0.55^{* *}$ & $0.55^{* *}$ \\
\hline 4. Figural fluency & 0.88 & $10.06(4.25)$ & 8.40 (3.99) & $10.91(4.12)^{* *}$ & & & & & $0.93^{* *}$ & $0.82 * *$ \\
\hline 5. Figural originality & 0.83 & $4.90(3.05)$ & $4.05(2.79)$ & $5.34(3.09)^{* *}$ & & & & & & $0.75^{* *}$ \\
\hline 6. Figural flexibility & 0.69 & $5.10(1.27)$ & 4.69 (1.39) & $5.31(1.15)^{* *}$ & & & & & & \\
\hline
\end{tabular}

a Statistical significance was determined by permutation testing. ${ }^{*} P<0.05,{ }^{*} P<0.01$.

analysis under three different genetic models (dominant, additive and recessive) was performed using linear regression in Plink. For SNP with minor allele homozygotes $<5 \%$, only the dominant model was tested. Pair-wise linkage disequilibrium (LD) and haplotype blocks were assessed by Haploview. Association analysis for the identified haplotype blocks was performed using linear regression in Plink. Haplotypes with estimated frequency $<5 \%$ were excluded from the analysis. For both single SNP and haplotype analysis, empirical $P$-values were computed by using the maxT permutation procedure implemented in Plink with 10,000 permutations.

Gene-gene interactions among genetic variants of COMT and DRD2 were analyzed using the Quantitative Multifactor Dimensionality Reduction (QMDR) approach implemented in MDR v3.0.2 software (Hahn et al., 2003). The QMDR approach is an extension of the original MDR approach to handle quantitative traits, and has been described in detail previously (Gui et al., 2013). In brief, the QMDR approach shared the same reduction strategy and cross-validation procedure as the MDR approach and the difference is that, the QMDR approach handles quantitative data by modifying MDR's constructive induction algorithm to use a $t$-test. In QMDR, by comparing the mean outcome of each multi-locus genotype combination to the overall mean outcome, all possible multi-locus genotype combinations were first combined into two different groups (high-level or low-level). Then the mean outcome of the high-level and the low-level group was compared using a $t$-test and the $t$-test statistics from the training set and testing set were defined as the scores to determine the best interaction model. In the present study, to reduce redundancy, SNPs in strong LD $\left(r^{2}>0.8\right)$ with another essayed SNP were first excluded, and then an exhaustive search of all possible two-, three- and four-way interactions among the remaining 18 SNPs (nine from COMT and nine from DRD2) was performed using QMDR. The best overall model was determined by 10 -fold cross-validation and the empirical $P$-values were computed by 1000 permutations.

\section{RESULTS}

Table 2 shows the descriptive statistics and inter-correlations matrix. All six DT scores demonstrated reliability and were highly inter-correlated. Female participants performed better in DT tests than male participants.

\begin{tabular}{|c|c|c|c|c|}
\hline SNPa & Position ${ }^{b}$ & Location & Allele (minor/major) & MAF (\%) \\
\hline rs737865 & 18310121 & Intron 1 & $\mathrm{C} / \mathrm{T}$ & 29.8 \\
\hline rs174675 & 18314051 & Intron 1 & $\mathrm{~T} / \mathrm{C}$ & 39.0 \\
\hline rs5993882 & 18317533 & Intron 1 & $\mathrm{G} / \mathrm{T}$ & 12.2 \\
\hline rs5993883 & 18317638 & Intron 1 & $\mathrm{G} / \mathrm{T}$ & 41.5 \\
\hline rs4646312 & 18328337 & Intron 1 & $\mathrm{C} / \mathrm{T}$ & 37.0 \\
\hline rs6269 & 18329952 & Exon 3 & $\mathrm{G} / \mathrm{A}$ & 37.4 \\
\hline rs4633 & 18330235 & Exon 3 & $\mathrm{~T} / \mathrm{C}$ & 26.2 \\
\hline rs6267 & 18330263 & Exon 3 & $\mathrm{~T} / \mathrm{G}$ & 7.5 \\
\hline rs4818 & 18331207 & Exon 4 & $\mathrm{G} / \mathrm{C}$ & 36.7 \\
\hline rs4680 & 18331271 & Exon 4 & $A / G$ & 26.7 \\
\hline rs769224 & 18331804 & Exon 5 & $A / G$ & 6.6 \\
\hline rs174697 & 18333832 & Intron 5 & $A / G$ & 33.4 \\
\hline
\end{tabular}

MAF, minor allele frequency. ${ }^{a}$ SNPs are listed down the column in sequential order from the $5^{\prime}$ end to the $3^{\prime}$ end of the sense strand of COMT. ${ }^{b}$ Physical position is based on NCBI Genome Build 36.3.

\section{SINGLE SNP AND HAPLOTYPE ASSOCIATION}

No significant deviations from Hardy-Weinberg equilibrium were observed for the 12 COMT SNPs (data not shown). Table 3 summarizes the results of single SNP analysis. In particular, for verbal DT test, rs174697 showed nominal association with verbal originality under the additive model. For figural DT test, two SNPs (rs737865 and rs5993883) were nominally associated with figural fluency under the dominant model, and two SNPs (rs737865 and rs4680) showed nominal associations with figural originality under the additive model. The LD patterns of the 12 COMT SNPs are shown in Figure 1. Two haplotype blocks were defined using the solid spine of LD algorithm, and were further tested for association with creative potential. The 3-SNP haplotype block (Block 1) displayed nominal association with figural originality. Specifically, the TCT and CCT haplotype (rs737865-rs174675-rs5993882) were nominally associated with figural originality. For the 7SNP haplotype block, although the global test did not reveal any association, the TATGCAG and CGCGGGA haplotype (rs4646312-rs6269-rs4633-rs6267-rs4818-rs4680-rs769224) were 
Table 3 | Selected results of single SNP analysis for creative potential ${ }^{\text {. }}$

\begin{tabular}{|c|c|c|c|c|c|c|c|c|c|c|c|c|}
\hline SNP & \multicolumn{4}{|c|}{ Verbal originality } & \multicolumn{4}{|c|}{ Figural fluency $^{c}$} & \multicolumn{4}{|c|}{ Figural originality ${ }^{d}$} \\
\hline rs174675 & Dominant & -0.093 & -1.04 & 0.299 & Recessive & -0.076 & -0.621 & 0.529 & Recessive & 0.096 & 0.782 & 0.435 \\
\hline rs5993882 & Dominant & -0.037 & -0.361 & 0.721 & Dominant & 0.013 & 0.124 & 0.898 & Dominant & 0.091 & 0.892 & 0.374 \\
\hline rs5993883 & Dominant & -0.067 & -0.743 & 0.460 & Dominant & 0.190 & 2.11 & 0.035 & Dominant & -0.153 & -1.70 & 0.092 \\
\hline rs4633 & Dominant & 0.034 & 0.391 & 0.697 & Recessive & -0.264 & -1.74 & 0.079 & Dominant & 0.159 & 1.85 & 0.065 \\
\hline rs6267 & Dominant & -0.117 & -0.951 & 0.339 & Dominant & -0.068 & -0.550 & 0.578 & Dominant & -0.002 & -0.01 & 0.986 \\
\hline rs4818 & Additive & 0.064 & 1.05 & 0.296 & Additive & 0.104 & 1.70 & 0.081 & Dominant & -0.159 & -1.83 & 0.066 \\
\hline rs4680 & Recessive & -0.022 & -0.149 & 0.882 & Recessive & -0.199 & -1.33 & 0.179 & Additive & 0.144 & 2.20 & 0.030 \\
\hline rs769224 & Dominant & 0.168 & 1.32 & 0.187 & Dominant & 0.027 & 0.214 & 0.835 & Dominant & 0.019 & 0.152 & 0.881 \\
\hline
\end{tabular}

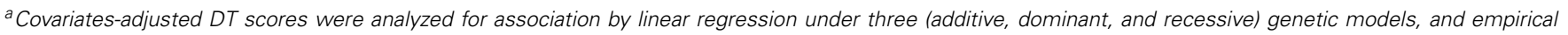

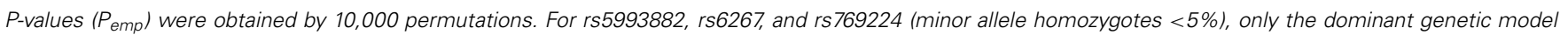
was tested. Only the best result and the corresponding genetic model are shown for each SNP. The significance values <0.05 are indicated in bold.

${ }^{b}$ Adjusted for gender and verbal fluency.

${ }^{c}$ Adjusted for gender.

${ }^{d}$ Adjusted for gender and figural fluency.

found to be nominally associated with figural originality and verbal flexibility, respectively, (Table 4). None of these nominal single SNP and haplotype associations withstood correction for multiple testing (data not shown).

\section{GENE-GENE INTERACTION}

Table 5 summarizes the results of QMDR analysis for DT verbal fluency, verbal flexibility, and figural flexibility. For verbal fluency, the overall best model was a four-way interaction of rs174675 (COMT), rs174697 (COMT), rs1076560 (DRD2), and rs4436578 (DRD2). These four SNPs had a training score of 8.60 and a testing score of 5.62. The cross-validation consistency of this model was 10/10. The 1000 permutation testing revealed a significant empirical $P$-value of 0.002 . For verbal flexibility, the overall best model was a four-way interaction of rs174675 (COMT), rs4818 (COMT), rs1076560 (DRD2) and rs4648317 (DRD2), with a training score of 7.97, a testing score of 4.76, and a cross-validation consistency of 10/10. Empirical $P$-value of this model was of 0.005 . For figural flexibility, the overall best model was a three-way interaction of rs5993883 (COMT), rs4648319 (DRD2), and rs4648317 (DRD2). This model showed a training score of 5.65 , a testing score of 3.97 , a cross-validation consistency of $10 / 10$, and an empirical $P$-value of 0.027 . No statistically significant interactions were reported for other DT scores.

\section{DISCUSSION}

Given the hypothesized role of DA in the neurobiological underpinnings of creativity as well as the crucial role of COMT in the regulation of DA transmission, COMT is among the many highly plausible functional candidate genes for creativity.
To date there have been only two published studies that have examined the association of COMT with creative potential. Both of these studies focused on rs4680. The rs 4680 is among one of the most frequently studied COMT polymorphisms. The G to A substitution at this position translates into a substitution of methionine (MET) for valine (VAL) at codon 158, which results in 3- to 4-fold difference in COMT enzymatic activity (Lotta et al., 1995; Lachman et al., 1996; Chen et al., 2004). The A allele has lower enzymatic activity than the $G$ allele, thereby leading to less efficient degradation of DA and higher DA level in the synaptic cleft (Chen et al., 2004). In a sample of 92 Caucasians, Reuter et al. (2006) first investigated the association of rs 4680 with creative potential, and reported no association of rs 4680 with creative potential. However, because Reuter et al. employed a composite index as a measure of creative potential, the particular contribution of rs 4680 to the three core dimensions of DT (fluency, originality, and flexibility) could not be determined. Runco et al. (2011) extended this work by examining the specific effect of rs 4680 on both verbal and figural DT fluency, originality and flexibility, and found rs4680 was associated with DT fluency. However, it should be noted that, both studies used small sample sizes, and neither of them examined other genetic variants of COMT.

The present study extended previous research by conducting a detailed association analysis of COMT with creative potential in a relatively large Chinese sample using 12 SNPs spanning the gene. Single SNP analysis identified four SNPs nominally associated with creative potential. Among the four SNPs, the previously reported rs4680 was found to be nominally associated with figural originality, with the A allele associated with higher figural originality score. This result is in line with previous findings that the A allele of rs4680 is associated with better creativity related 


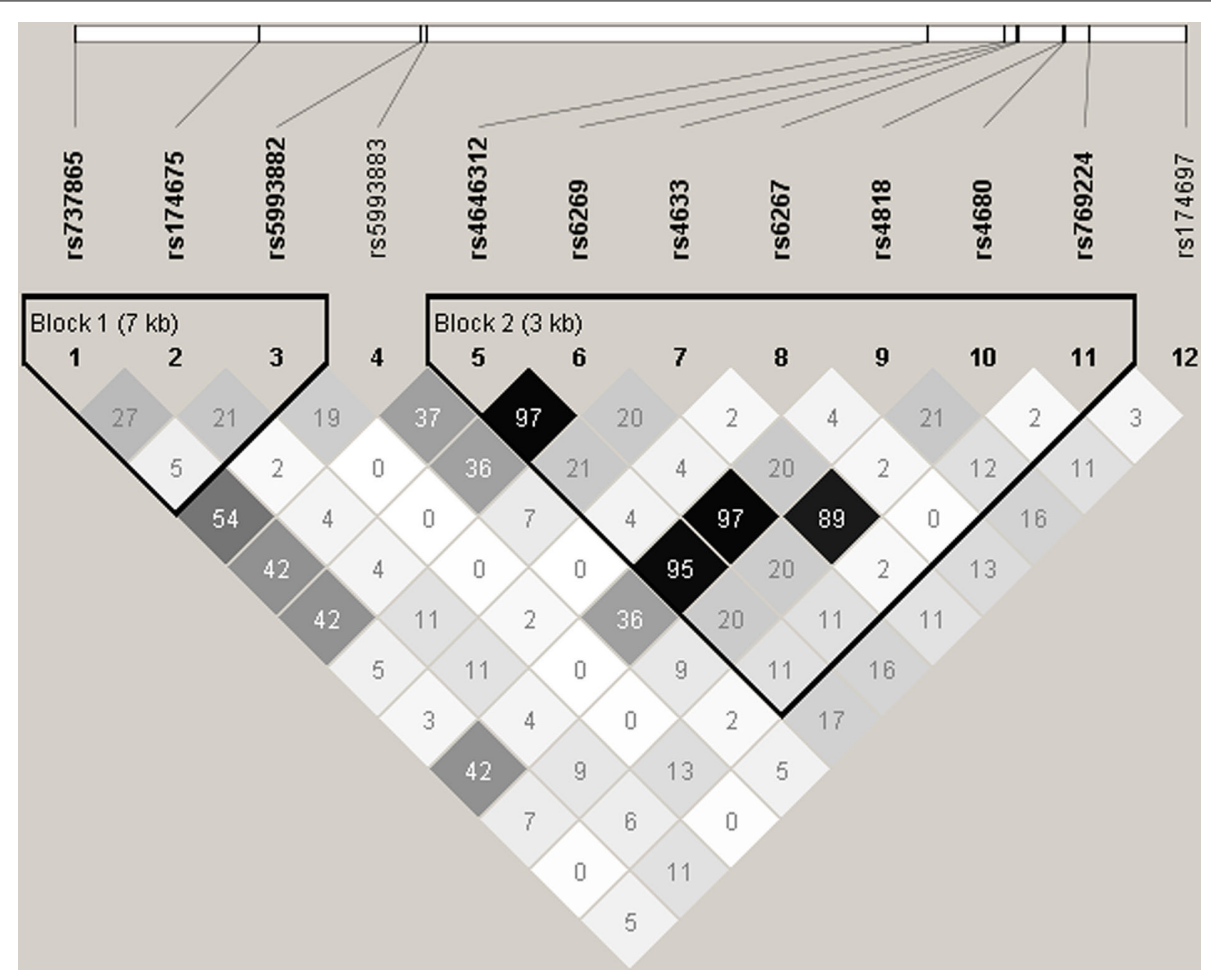

FIGURE 1 | Linkage disequilibrium (LD) pattern of the 12 COMT SNPs analyzed in the present study. Numbers in squares designate the degree of LD ( $r^{2}$ ) between any two SNPs. LD blocks were defined using the solid spine of LD algorithm implemented in Haploview.

cognitive performance (such as working memory) (Egan et al., 2001; Malhotra et al., 2002; Bruder et al., 2005; Barnett et al., 2007; Aguilera et al., 2008; Diaz-Asper et al., 2008) and creativity related personality traits (such as novelty seeking) (Tsai et al., 2004; Golimbet et al., 2007; Davila et al., 2013). However, it is also important to note that, the previously reported association of rs4680 with DT fluency was not replicated in the present study. One possible explanation for this discrepancy could be the genetic heterogeneity between Han Chinese and Caucasians. Besides rs4680, three intronic SNPs also showed nominal associations with creative potential. The rs737865 was nominally associated with both figural fluency and figural originality, while rs5993883 and rs174697 were nominally associated with figural fluency and verbal originality, respectively. For these intronic SNPs, because few studies have been conducted to examine their functions, there exists less supporting evidence for the observed associations. However, previous research has shown that intronic variants may play critical roles in the regulation of gene expression (Nackley et al., 2006; Wang and Cooper, 2007; Zhang et al., 2007). It is possible that these intronic SNPs might play a role in regulating COMT expression. Further functional analysis is needed to test this hypothesis. Another possibility is that these SNPs might be in high LD with other unidentified causative variants. In addition to single SNP analysis, haplotype analysis also identified three haplotypes (the TCT and CCT haplotype of Block1, and the TATGCAG haplotype of Block 2) nominally associated with figural originality, as well as one haplotype (the CGCGGGA haplotype of Block 2) nominally associated with verbal flexibility. However, caution should be exercised when interpreting these results, since these nominally significant single SNP and haplotype associations would not survive correction for multiple testing. Therefore, these results should only be considered as suggestive, replication by independent studies is necessary to confirm these nominal findings.

The nonlinear relationship between DA and creative potential suggests that genes involved in DA transmission may interact to affect creative potential (Chermahini and Hommel, 2010, 2012). Our previous study found some suggestive evidence for the association of DRD2 with creative potential. Therefore, the present study further extended our previous study by systematically exploring the interaction between COMT and DRD2. For these two genes, a total of 18 SNPs were included and examined for all their possible two-, three- and four-way interactions using the QMDR approach. Linear regression or MANOVA is commonly used to detect statistical interactions, however, these traditional parametric statistical methods are less practical for genetic studies attempting to examine possible interactions of multiple genetic variants, for several reasons. First, when high-order interactions involving high-dimensional data are considered, there may be many sparse or empty cells, resulting in inaccurate parameter estimates and an increased type I error. Second, as each additional main effect is included in the model, the total number of parameters (especially for interaction terms) grows exponentially, resulting in too many degrees of freedom and increased type I 
Table 4 | Selected results of haplotype analysis for creative potential ${ }^{\mathrm{a}}$.

\begin{tabular}{|c|c|c|c|c|c|c|c|c|}
\hline Block & Haplotype & Frequencies (\%) & \multicolumn{3}{|c|}{ Verbal flexibility $^{d}$} & \multicolumn{3}{|c|}{ Figural originality } \\
\hline & ССТ & 29.7 & 0.064 & 0.956 & 0.323 & -0.163 & 6.23 & 0.014 \\
\hline & $\mathrm{TTT}$ & 26.8 & -0.005 & 0.006 & 0.939 & -0.029 & 0.175 & 0.679 \\
\hline & TTG & 12.2 & -0.053 & 0.316 & 0.577 & 0.105 & 1.27 & 0.265 \\
\hline \multirow[t]{5}{*}{ Block $2^{\mathrm{c}}$} & CGCGGGG & 29.7 & 0.031 & 0.211 & 0.647 & -0.122 & 3.31 & 0.068 \\
\hline & TACGCGG & 28 & 0.030 & 0.212 & 0.642 & -0.029 & 0.200 & 0.650 \\
\hline & TATGCAG & 25.3 & 0.085 & 1.64 & 0.204 & 0.140 & 4.41 & 0.039 \\
\hline & TACTCGG & 7.5 & -0.092 & 0.655 & 0.419 & 0.019 & 0.029 & 0.868 \\
\hline & CGCGGGA & 6.6 & -0.249 & 4.03 & 0.042 & 0.029 & 0.054 & 0.820 \\
\hline
\end{tabular}

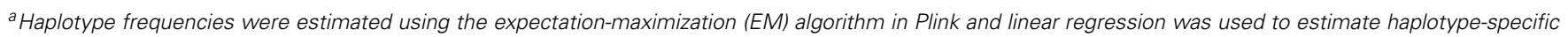

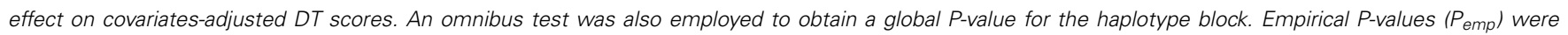

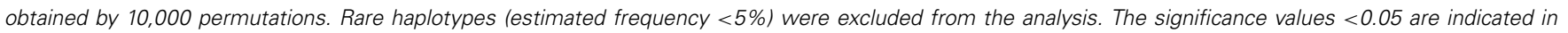
bold.

${ }^{b}$ The order of SNPS in block 1 was rs737865, rs174675, and rs5993882.

${ }^{c}$ The order of SNPs in block 2 was rs4646312, rs6269, rs4633, rs6267, rs4818, rs4680, and rs769224.

${ }^{d}$ Adjusted for gender and verbal fluency.

eAdjusted for gender and figural fluency.

Table 5 | Selected results of gene-gene interaction analysis for creative potential ${ }^{\mathrm{a}}$.

\begin{tabular}{|c|c|c|c|c|c|}
\hline DT score & Combination & CVC & Training score & Testing score & $P_{e m p}$ \\
\hline Verbal fluency & rs174675 (COMT), rs174697 (COMT), rs1076560 (DRD2) & $3 / 10$ & 5.53 & -0.971 & 0.943 \\
\hline \multirow[t]{2}{*}{ Verbal flexibility ${ }^{\mathrm{C}}$} & rs4818 (COMT), rs1076560 (DRD2) & $4 / 10$ & 3.83 & -0.427 & 0.871 \\
\hline & rs4818 (COMT), rs1076560 (DRD2), rs4648317 (DRD2) & $3 / 10$ & 5.58 & 0.368 & 0.688 \\
\hline \multirow[t]{3}{*}{ Figural flexibility ${ }^{d}$} & rs174675 (COMT), rs6279(DRD2) & $4 / 10$ & 3.45 & -0.637 & 0.906 \\
\hline & rs5993883 (COMT), rs4648319 (DRD2), rs4648317 (DRD2) & $10 / 10$ & 5.65 & 3.97 & 0.027 \\
\hline & rs5993883 (COMT), rs174697 (COMT), rs4648319 (DRD2), rs4648317 (DRD2) & $7 / 10$ & 7.67 & 0.987 & 0.500 \\
\hline
\end{tabular}

CVC, cross-validation consistency.

a Gene-gene interaction analysis for covariates-adjusted DT scores was performed by QMDR. An exhaustive search of all possible two-, three- and four-way interactions was performed among the 18 COMT and DRD2 SNPs. For COMT, the SNPs included were rs737865, rs174675, rs5993882, rs5993883, rs6267, rs4818, rs4680, rs769224, and rs174697. For DRD2, the SNPs included were rs6279, rs6277, rs 1076560, rs4436578, rs4648319, rs4245148, rs4648317, rs 1799732, and rs1799978. Empirical P-values ( $\left.P_{\text {emp }}\right)$ were obtained by 1000 permutations. The significance values $<0.05$ are indicated in bold.

${ }^{b}$ Adjusted for gender.

${ }^{c}$ Adjusted for gender and verbal fluency.

${ }^{d}$ Adjusted for gender and figural fluency.

and type II errors. Third, the lack of a simple pattern of dominant, additive or recessive effect of alleles for common complex traits makes it nearly impossible to model the interaction terms. To address these issues, the non-parametric QMDR approach was proposed. The major advantage of QMDR is that, by effectively reducing the combinations of multi-locus genotypes from high dimensions to one dimension, it avoids the issues of sparse data cells and greatly reduces the degree of freedom necessary for modeling higher-order interactions that can cause problems for traditional parametric statistical methods, thus facilitating the simultaneous detection and characterization of multi-locus interactions and retaining reasonable power even with relatively small sample size. In addition, QMDR does not require any assumption on genetic model, thus it can be more useful to study complex 
trait, in which the mode of genetic inheritance is usually unknown a prior.

Employing the QMDR approach, the present study identified one significant four-way interaction model (rs174675, rs174697, rs1076560, and rs4436578) associated with verbal fluency, one significant four-way interaction model (rs174675, rs4818, rs1076560, and rs4648317) associated with verbal flexibility, as well as one significant three-way interaction model (rs5993883, rs4648319, and rs4648317) associated with figural flexibility. Because QMDR combines both cross-validation testing and permutation testing to select the best model from all possible two-, three- and four-way models, the problem of multiple testing that inflates the type I error rate in single SNP and haplotype analysis does not apply to the results of QMDR analysis. These three interaction models therefore provide more convincing evidence that COMT and DRD2 are involved in creative potential and act in coordination to contribute to creative potential. In addition, it is worth noting that, among SNPs involved in these three interaction models, only $D R D 2$ rs 1076560 showed a nominal main effect on verbal fluency (Zhang et al., 2014), other SNPs did not demonstrate a main effect on the corresponding DT scores in single SNP analysis. Therefore, this finding further suggests that the underlying genetic mechanism of creativity might be complex and genetic variants without main effect may also contribute to creativity by their interaction effects.

It is intriguing to note that, although both COMT and DRD2 are key genes involved in DA transmission, they are likely to be related to different DA pathways: COMT to the prefrontal pathway whereas $D R D 2$ to the striatal pathway. Accumulating evidence has suggested that these two different pathways may subserve distinct cognitive processes, with DA in the PFC promoting cognitive stability by increasing distractor resistance, while conversely, DA in the striatum promoting cognitive flexibility by allowing the updating of newly relevant representations (Cools and D'Esposito, 2009). Interestingly, although cognitive stability and flexibility are functionally opposed, according to the recent dual-pathway model of creativity, both of them contribute to creativity and creative performance arises from the interaction between them (De Dreu et al., 2008; Nijstad et al., 2010). The model suggests that, while cognitive flexibility facilitates creative potential by establishing more categories of idea and thus contributing to flexibility and fluency, cognitive stability also promotes creative potential by generating many ideas within a few categories and thus contributing to fluency. And most importantly, in the course of ideation people may dynamically employ both the stable processing mode and the flexible processing mode. Based on this notion, it is reasonable to expect that the observed interaction between COMT and DRD2 may implicate individual differences in regulating the dynamic interplay between prefrontal and striatal networks during ideation. Individuals with different combinations of COMT and DRD2 genotypes may vary in the degree to which the stable processing mode and the flexible processing mode are used as well as the patterns how the dynamic balance between these two processing modes is modulated, thus resulting in differences in ideation fluency and flexibility. However, given that the biological functions of the majority of the SNPs included in these interaction models are largely unknown, such explanation remains highly speculative. The specific mechanisms by which these interactions actually work cannot be elucidated in the present study. Further studies integrating both functional analysis and cognitive neuroscience approach will help clarify the detailed biological mechanisms of these interactions.

There are limitations to the present study. First, although the largest such study to date, the moderate sample size of the present study is not as large as would be ideal. Furthermore, since both allele frequencies and LD patterns vary greatly across ethnic populations and only one ethnic group was examined in the present study, the generalization of these findings to other populations is limited. Thus, further prospective studies using larger sample size and replication studies in other ethnic populations are warranted to confirm these suggestive findings. Second, although three significant interaction models between COMT and DRD2 were identified in the present study, sorting out the nature of the interactions in high-dimensional space to infer function remains an interpretive challenge. Functional analysis of these genotypic combinations would be needed to help elucidate these effects. Third, other crucial genes in DA transmission, such as DRD4 (Mayseless et al., 2013) and DAT, were not examined in the present study. Since the regulation of DA transmission is a complex network involving multiple process, full genetic contribution of DA related variants to creativity will rely on far more complex interactions of multiple DA receptors (e.g., $D R D 1, D R D 2$, and $D R D 4$ ), transporters and enzymatic polymorphisms (e.g., $D A T, C O M T, M A O A$, and $M A O B)$. Further studies systematically involving such interactions are needed to obtain a clearer overview of DA transmission.

In conclusion, the present study provides preliminary evidence for the involvement of COMT as well as the interaction between $C O M T$ and $D R D 2$ in creative potential. Although the underlying mechanisms still need to be further investigated, this exploratory study may provide important information to elucidate the contribution of DA related genes to creative potential, which will undoubtedly lead to a better understanding of the underlying genetic basis of creativity.

\section{AUTHOR CONTRIBUTIONS}

Shun Zhang and Jinghuan Zhang were involved in the conception and design of the work. Shun Zhang and Muzi Zhang were involved in data collection. Shun Zhang analyzed the data. Shun Zhang and Jinghuan Zhang contributed in writing the main manuscript text.

\section{ACKNOWLEDGMENTS}

This research was funded by the key projects of Philosophy and Social Science Grant of Department of Education, China (11JZD040), Humanities and Social Science Planning Grant of Department of Education, China (12YJA190024), Science and Technology Planning Projects of Shandong Province, China (2012GGB01189), and Key Subject Funds of Shandong Province, China (2011-2015). We appreciate Dr. Mark A. Runco (Torrance Creativity Center, University of Georgia) for the directions and help about divergent thinking tests scoring. We appreciate Dr. Jason H. Moore and Dr. Peter Andrews (Institute for 
Quantitative Biomedical Sciences, Geisel School of Medicine) for their help with QMDR. We appreciate Dr. Zbigniew R. Struzik and two reviewers for their very constructive comments and advice.

\section{REFERENCES}

Aguilera, M., Barrantes-Vidal, N., Arias, B., Moya, J., Villa, H., Ibanez, M. I., et al. (2008). Putative role of the COMT gene polymorphism (Val158Met) on verbal working memory functioning in a healthy population. Am. J. Med. Genet. B Neuropsychiatr. Genet. 147B, 898-902. doi: 10.1002/ajmg.b. 30705

Axelrod, J. (1957). O-methylation of epinephrine and other catechols in vitro and in vivo. Science 126, 400-401. doi: 10.1126/science.126.3270.400

Barnett, J. H., Jones, P. B., Robbins, T. W., and Muller, U. (2007). Effects of the catechol-O-methyltransferase Val158Met polymorphism on executive function: a meta-analysis of the Wisconsin Card Sort Test in schizophrenia and healthy controls. Mol. Psychiatry 12, 502-509. doi: 10.1038/sj.mp. 4001973

Barrett, J. C., Fry, B., Maller, J., and Daly, M. J. (2005). Haploview: analysis and visualization of LD and haplotype maps. Bioinformatics 21, 263-265. doi: 10.1093/bioinformatics/bth457

Bertolino, A., Taurisano, P., Pisciotta, N. M., Blasi, G., Fazio, L., Romano, R., et al. (2010). Genetically determined measures of striatal D2 signaling predict prefrontal activity during working memory performance. PLoS ONE 5:e9348. doi: 10.1371/journal.pone.0009348

Bruder, G. E., Keilp, J. G., Xu, H., Shikhman, M., Schori, E., Gorman, J. M., et al. (2005). Catechol-O-methyltransferase (COMT) genotypes and working memory: associations with differing cognitive operations. Biol. Psychiatry. 58, 901-907. doi: 10.1016/j.biopsych.2005.05.010

Chen, J., Lipska, B. K., Halim, N., Ma, Q. D., Matsumoto, M., Melhem, S., et al. (2004). Functional analysis of genetic variation in catechol-O-methyltransferase (COMT): effects on mRNA, protein, and enzyme activity in postmortem human brain. Am. J. Hum. Genet. 75, 807-821. doi: 10.1086/425589

Chermahini, S. A., and Hommel, B. (2010). The (b)link between creativity and dopamine: spontaneous eye blink rates predict and dissociate divergent and convergent thinking. Cognition 115, 458-465. doi: 10.1016/i.cognition.2010.03.007

Chermahini, S. A., and Hommel, B. (2012). More creative through positive mood? Not everyone! Front. Hum. Neurosci. 6:319. doi: 10.3389/fnhum.2012.00319

Colzato, L. S., Van Den Wildenberg, W. P., and Hommel, B. (2013). Cognitive control and the COMT ValMet polymorphism: genetic modulation of videogame training and transfer to task-switching efficiency. Psychol. Res. doi: 10.1007/s00426-013-0514-8

Colzato, L. S., Waszak, F., Nieuwenhuis, S., Posthuma, D., and Hommel, B. (2010). The flexible mind is associated with the catechol-O-methyltransferase (COMT) Val158Met polymorphism: evidence for a role of dopamine in the control of task-switching. Neuropsychologia 48, 2764-2768. doi: 10.1016/j.neuropsychologia.2010.04.023

Cools, R., and D’Esposito, M. (2009). "Dopaminergic modulation of flexible cognitive control in humans," in Dopamine Handbook, eds A. Björklund, S. Dunnet, L. Iversen, and S. Iversen. (Oxford: Oxford University Press), 249-260.

Davila, W., Basterreche, N., Arrue, A., Zamalloa, M. I., Gordo, E., Davila, R., et al. (2013). The influence of the Val158Met catechol-O-methyltransferase polymorphism on the personality traits of bipolar patients. PLOS ONE 8:e62900. doi: 10.1371/journal.pone.0062900

De Dreu, C. K. W., Baas, M., and Nijstad, B. A. (2008). Hedonic tone and activation level in the mood-creativity link: toward a dual pathway to creativity model. J. Pers. Soc. Psychol. 94, 739-756. doi: 10.1037/0022-3514.94.5.739

Diaz-Asper, C. M., Goldberg, T. E., Kolachana, B. S., Straub, R. E., Egan, M. F., and Weinberger, D. R. (2008). Genetic variation in catechol-O-methyltransferase: effects on working memory in schizophrenic patients, their siblings, and healthy controls. Biol. Psychiatry 63, 72-79. doi: 10.1016/j.biopsych.2007.03.031

Egan, M. F., Goldberg, T. E., Kolachana, B. S., Callicott, J. H., Mazzanti, C. M., Straub, R. E., et al. (2001). Effect of COMT Val108/158 Met genotype on frontal lobe function and risk for schizophrenia. Proc. Natl. Acad. Sci. U.S.A. 98, 6917-6922. doi: 10.1073/pnas.111134598

Flaherty, A. W. (2005). Frontotemporal and dopaminergic control of idea generation and creative drive. J. Comp. Neurol. 493, 147-153. doi: 10.1002/cne.20768
Golimbet, V. E., Alfimova, M. V., Gritsenko, I. K., and Ebstein, R. P. (2007). Relationship between dopamine system genes and extraversion and novelty seeking. Neurosci. Behav. Physiol. 37, 601-606. doi: 10.1007/s11055-0070058-8

Gui, J., Moore, J. H., Williams, S. M., Andrews, P., Hillege, H. L., Van Der Harst, P., et al. (2013). A simple and computationally efficient approach to multifactor dimensionality reduction analysis of gene-gene interactions for quantitative traits. PLoS ONE 8:e66545. doi: 10.1371/journal.pone.0066545

Hahn, L. W., Ritchie, M. D., and Moore, J. H. (2003). Multifactor dimensionality reduction software for detecting gene-gene and gene-environment interactions. Bioinformatics 19, 376-382. doi: 10.1093/bioinformatics/btf869

Hocevar, D. (1980). Intelligence, divergent thinking, and creativity. Intelligence 4, 25-40. doi: 10.1016/0160-2896(80)90004-5

Lachman, H. M., Papolos, D. F., Saito, T., Yu, Y. M., Szumlanski, C. L., and Weinshilboum, R. M. (1996). Human catechol-O-methyltransferase pharmacogenetics: description of a functional polymorphism and its potential application to neuropsychiatric disorders. Pharmacogenetics 6, 243-250. doi: 10.1097/00008571-199606000-00007

Lotta, T., Vidgren, J., Tilgmann, C., Ulmanen, I., Melen, K., Julkunen, I., et al. (1995). Kinetics of human soluble and membrane-bound catechol Omethyltransferase: a revised mechanism and description of the thermolabile variant of the enzyme. Biochemistry 34, 4202-4210. doi: 10.1021/bi00013a008

Malhotra, A. K., Kestler, L. J., Mazzanti, C., Bates, J. A., Goldberg, T., and Goldman, D. (2002). A functional polymorphism in the COMT gene and performance on a test of prefrontal cognition. Am. J. Psychiatry 159, 652-654. doi: 10.1176/appi.ajp.159.4.652

Mayseless, N., Uzefovsky, F., Shalev, I., Ebstein, R. P., and Shamay-Tsoory, S. G. (2013). The association between creativity and 7R polymorphism in the dopamine receptor D4 gene (DRD4). Front. Hum. Neurosci. 7:502. doi: 10.3389/fnhum.2013.00502

Murphy, M., Runco, M. A., Acar, S., and Reiter-Palmon, R. (2013). Reanalysis of genetic data and rethinking dopamine's relationship with creativity. Creat. Res. J. 21, 147-148. doi: 10.1080/10400419.2013.752305

Nackley, A. G., Shabalina, S. A., Tchivileva, I. E., Satterfield, K., Korchynskyi, O., Makarov, S. S., et al. (2006). Human catechol-O-methyltransferase haplotypes modulate protein expression by altering mRNA secondary structure. Science 314, 1930-1933. doi: 10.1126/science.1131262

Nijstad, B. A., De Dreu, C. K. W., Rietzschel, E. F., and Baas, M. (2010). The dual pathway to creativity model: creative ideation as a function of flexibility and persistence. Eur. Rev. Soc. Psychol. 21, 34-77. doi: 10.1080/10463281003 765323

Purcell, S., Neale, B., Todd-Brown, K., Thomas, L., Ferreira, M. A., Bender, D., et al. (2007). PLINK: a tool set for whole-genome association and population-based linkage analyses. Am. J. Hum. Genet. 81, 559-575. doi: 10.1086/519795

Reuter, M., Roth, S., Holve, K., and Hennig, J. (2006). Identification of first candidate genes for creativity: a pilot study. Brain. Res. 1069, 190-197. doi: 10.1016/j.brainres.2005.11.046

Runco, M. A., and Albert, R. S. (1985). The reliability and validity of ideational originality in the divergent thinking of academically gifted and nongifted children. Educ. Psychol. Meas. 45, 483-501. doi: 10.1177/001316448504500306

Runco, M. A., Noble, E. P., Reiter-Palmon, R., Acar, S., Ritchie, T., and Yurkovich, J. M. (2011). The genetic basis of creativity and ideational fluency. Creat. Res. J. 23, 376-380. doi: 10.1080/10400419.2011.621859

Sternberg, R. J., and Lubart, T. I. (1999). "The concept of creativity: prospects and paradigms," in Handbook of Creativity, ed R. J. Sternberg (New York, NY: Cambridge University Press), 3-15.

Takeuchi, H., Taki, Y., Sassa, Y., Hashizume, H., Sekiguchi, A., Fukushima, A., et al. (2010). Regional gray matter volume of dopaminergic system associate with creativity: evidence from voxel-based morphometry. Neuroimage 51, 578-585. doi: 10.1016/j.neuroimage.2010.02.078

Tsai, S. J., Hong, C. J., Yu, Y. W., and Chen, T. J. (2004). Association study of catechol-O-methyltransferase gene and dopamine D4 receptor gene polymorphisms and personality traits in healthy young chinese females. Neuropsychobiology 50, 153-156. doi: 10.1159/000079107

Wallach, M. A., and Kogan, N. (1965). Modes of Thinking in Young Children. New York, NY: Holt, Rinehart and Wilson.

Wang, G. S., and Cooper, T. A. (2007). Splicing in disease: disruption of the splicing code and the decoding machinery. Nat. Rev. Genet. 8, 749-761. doi: $10.1038 / \operatorname{nrg} 2164$ 
Zhang, S., Zhang, M. Z., and Zhang, J. H. (2014). An exploratory study on DRD2 and creative potential. Creat. Res. J. 26, 115-123. doi: 10.1080/10400419.2014.874267

Zhang, Y., Bertolino, A., Fazio, L., Blasi, G., Rampino, A., Romano, R., et al. (2007). Polymorphisms in human dopamine D2 receptor gene affect gene expression, splicing, and neuronal activity during working memory. Proc. Natl. Acad. Sci. U.S.A. 104, 20552-20557. doi: 10.1073/pnas.0707 106104

Conflict of Interest Statement: The authors declare that the research was conducted in the absence of any commercial or financial relationships that could be construed as a potential conflict of interest.
Received: 26 December 2013; paper pending published: 06 February 2014; accepted: 27 March 2014; published online: 14 April 2014.

Citation: Zhang S, Zhang M and Zhang J (2014) Association of COMT and COMTDRD2 interaction with creative potential. Front. Hum. Neurosci. 8:216. doi: 10.3389/ fnhum.2014.00216

This article was submitted to the journal Frontiers in Human Neuroscience.

Copyright (c) 2014 Zhang, Zhang and Zhang. This is an open-access article distributed under the terms of the Creative Commons Attribution License (CC BY). The use, distribution or reproduction in other forums is permitted, provided the original author(s) or licensor are credited and that the original publication in this journal is cited, in accordance with accepted academic practice. No use, distribution or reproduction is permitted which does not comply with these terms. 